\title{
Umbilical Cord Arterial Blood Gas Study and Cord Blood Lactate Predictability for Unfavorable Neonatal Outcomes
}

\author{
E Eltaieb $^{1^{*}}$ and H Elkholy ${ }^{2}$ \\ ${ }^{1}$ Department of Obstetrics and Gynecology, Ain Shams University, Cairo, Egypt \\ ${ }^{2}$ Department of Pediatrics and Neonatology, Ain Shams University, Cairo, Egypt
}

Received: 盋 April 12, 2018; Published: 畊 April 18, 2018

*Corresponding author: E Eltaieb, Department of Obstetrics and Gynecology, Ain Shams University, Cairo, Egypt Tel: +2 01004711414;

Email: profobgyn81@hotmail.com

\begin{abstract}
Background: Prenatal asphyxia is a cornerstone issue influencing neonatal morbidity and mortality with long term health impact. Foetal electronic monitoring is clinically performed in a routine manner to reflect the fetal clinical status throughout intra partum period of time. Normal cardiotocography trace is in powerful correlation with absent academia occurrence at delivery of the neonate. Abnormality of cardiotocographic trace on the other hand trace requires extra clinical evaluation and research implementation. To reflect the metabolic pathways of the neonate, umbilical artery lactate have been analyzed and evaluated. Aim of this research study is to verify the clinical power and value of umbilical cord lactate level in predictability of the unfavorable early onset neonatal outcomes in clinical scenarios of fetal distress in intra partum period and to statistically compare and contrast the clinical value of umbilical cord lactate and umbilical artery $\mathrm{pH}$ in predictability undesirable neonatal clinical outcomes having intra partum foetal distress issues.
\end{abstract}

Methodology: 295 gestations with abnormalities of cardiotocographic traces in active stage of labor have undergone analysis and measurement of umbilical cord blood lactate levels and umbilical artery pH measurements straight away after birth and have been compared statistically for predictability of unfavorable newborn outcomes.

Results: In the current research study statistically calculated specificity of cord serum lactate (97.7\%) and umbilical cord arterial pH (95.97\%) was displayed clearly to be almost similar in neonates with Apgar scores $<7$ at 1 minute and 5 minutes, but the statistically obtained sensitivity of umbilical cord serum lactate levels (23.14\%) and umbilical cord ph (31.4\%) was less in neonates with Apgar scores $\leq 7$ at 1 minute. Area under ROC displayed and demonstrated that serum lactate levels are much more precise in forecasting unfavorable neonatal outcomes in comparison to umbilical cord artery $\mathrm{pH}$.

Conclusion: Umbilical blood lactate levels are more statistically specific than umbilical artery pH measurements in forecasting unfavorable neonatal clinical outcomes. The area under ROC curve (at 95th percentile) have displayed that serum lactate levels is more precise in forecasting unfavorable newborn clinical outcomes in comparison to umbilical cord artery pH at delivery.

\section{Introduction}

Labor is a physiologically stressful situation experience for the fetus. Foetal distress is clinically described as "a situation where physiological pathways and events are manipulated in a manner making mortality or everlasting injurious insult a possibility within a fairly short zone of time and frequently implies interference of normal fetal physiological oxygenation pathway, varying from trivial hypoxic issues to severe foetal asphyxia"[1,2]. Extensive research studies have been performed to explore and discriminate

risk factors and responsibility of asphyxia in intra partum period leading consequently unfavorable neonatal clinical outcomes when delivered at full term gestational age $[3,4]$. The goal of clinical foetal monitoring is to efficiently identify fetal risks for neonatal and long-term insults due to intra partum asphyxia in order to avoid efficiently neonatal morbidity and mortality. Clinical classic tools implemented for intra partum foetal monitoring are intermittent pattern of foetal heart auscultation, CTG and foetal scalp blood ph analysis [4,5]. 
CTG surveillance is an updated and clinically non-invasive tool for evaluating together ante partum and intra partum fetal clinical status, this tool shows a significant clinical impact on obstetrical practice. 4

Intra partum surviellance tool with great precision is needed to make sure that optimal foetal well-being in the physiological process of labor. Intermittent FHR auscultation and foetal electronic surveillance e.g CTG are the chief tools clinically implemented for intra partum foetal monitoring; the parameters integrated in these tools involve basal heart rate, variability, accelerations and decelerations [6,7]. However despite popularity of clinical application of Cardiotocographic is not a perfect tool reflecting fetal well being l. Despite the fact that a normal cardiotocographic trace is foretelling of a normal acid base physiological status at delivery, however an abnormal cardiotocographic trace have a low positive predictive value as regards fetal $\mathrm{pH}<7.25$.6. After delivery occurrence intrauterine foetal asphyxia is clinically assessed in a subjective manner by APGAR scoring and in an objective manner by neonatal cord blood $\mathrm{pH}$ and Lactate measurements.

High lactate levels are interrelated to foetal metabolic anaerobic glycolysis pathway occurring in oxygen deprived fetal tissues. Umbilical artery $\mathrm{pH}$ and lactate measurements are considered as an effective and precise method for diagnosing of fetal intra partum distress [8,9]. The accurate incidence of foetal distress is vague, but estimated to range from one in every 25 deliveries till one in every 100 deliveries. Normal CTG trace correlate greatly with nonexistence of academia issues at delivery. Abnormalities of CTG traces require additional assessment. Therefore this research study is performed to explore the strength of CTG in association with umbilical cord arterial blood gas assessment and cord lactate levels at birth. Changes in the umbilical cord blood $\mathrm{pH}$ and lactate is correlated to the level of foetal distress and foetal buffering physiological capacity. This research permits comparison of cardiotocographic abnormalities and umbilical cord arterial blood gas analysis and cord blood lactate level in uncovering of neonatal metabolic condition after delivery [10-12].

\section{Methods}

A research study conducted in an observational manner on 295 gestations in labor with CTG abnormalities in Obstetrics and Gynecology department in Ain Shams Maternity Hospital. The following research criteria were implemented for subject recruitment.

Inclusive Research Criteria: > 37 weeks gestational age singleton gestations with vertex presentation in active labor with fetal distress.

Exclusive Research Criteria: High-risk gestations (anemia, hypertensive, thyroid dysfunctions, DM, epileptic, asthmatic), Intrauterine growth retardation, oligohydromnios, premature deliveries, prior caesarean delivery, elective caesarean delivery, multifetal pregnancies, mal presentations, placental abruption, fetal congenital anomalies, Maternal infections and PROM . Research conducted from July 2015 to July 2017. The research was explained to the recruited study subjects in antenatal care clinics and during the time of labor, CTG was performed for all subjects. After birth of the baby immediately, umbilical cord arterial blood was sampled and analyzed for lactate and cord blood $\mathrm{pH}$ with cardiotocographic trace abnormalities. The neonate was clinically observed and assessed for 24 hours to evaluate wellbeing and unfavorable outcomes. Consent was obtained from all study subjects recruited meeting inclusive research criteria, CTG was performed during intra partum phase, 2 blood samples were obtained at once after birth by double clamping the umbilical cord in neonates with CTG abnormalities. Umbilical cord blood was sampled from an isolated portion into a heparinised syringe for blood gas analysis. Additionally $2 \mathrm{ml}$ of cord arterial blood sample was kept into sodium fluoride potassium oxalate tube. In order to measure lactate levels and umbilical cord arterial blood gas analysis. Unfavorable early neonatal clinical outcomes e.g low Apgar scores, NICU admissions, assisted ventilation like bag and mask, intubation, in first 24 hours after delivery were compared with lactate levels and arterial blood gas analysis.

The following parameters were considered in the research findings analysis:

\section{Umbilical Artery pH 1}

$<7.15$ : neonatal Acidemia

$<7$ : Severe neonatal acidemia

\section{Cord Blood Lactate 29}

$>$ 5mol/l: neonatal acidemia

$>10 \mathrm{mmol} / \mathrm{l}$ : Severe neonatal academia

To statistically analyze which test is superior, ROC curve was made by performing sensitivity and specificity for various parameters. Precision was calculated by measuring the area under the ROC curve.

An area of 1 represents a perfect test; an area of 5 represents a worthless test. A rough guide for classifying the accuracy of a diagnostic test is the traditional academic point system:

$$
\begin{aligned}
& 0.90-1=\text { excellent }(A) \\
& 0.80-0.90=\operatorname{good}(B) \\
& 0.70-0.80=\text { fair }(C) \\
& 0.60-0.70=\text { poor }(D) \\
& 0.50-0.60=\text { fail }(F)
\end{aligned}
$$

Statistical package for social services (SPSS) was used for analysis. 


\section{Results}

The area under ROC curve displays that serum lactate levels are much more precise in predictability of unfavorable Prenatal clinical outcomes in comparison to umbilical artery $\mathrm{pH}$. The calculated sensitivity and specificity for lactate levels and $\mathrm{pH}$ measurements in correlation to NICU admission, Resuscitation, low Apgar scoring at $1 \mathrm{~min}, 5 \mathrm{~min}$ and clinical outcome are displayed as receiveroperator curves.

\section{ROC for Resuscitation}

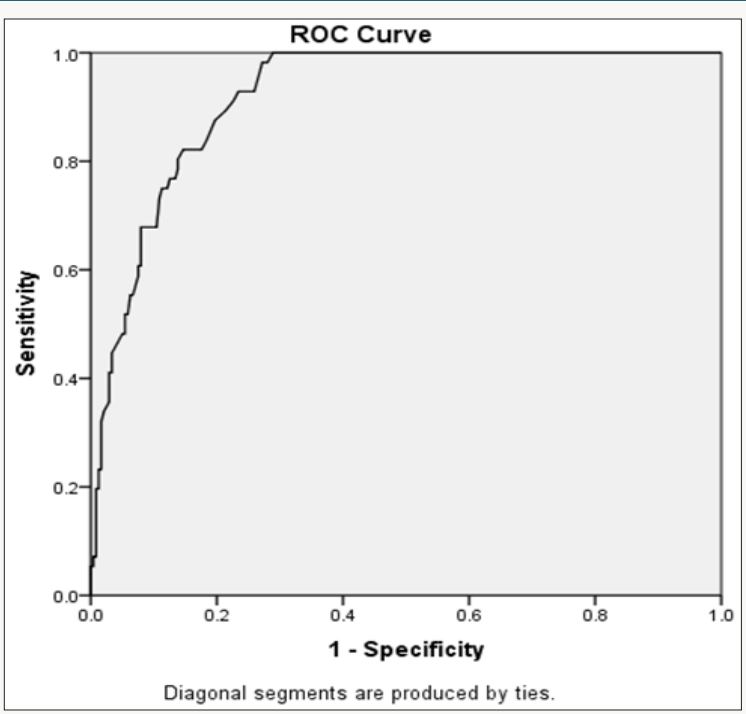

Figure 1: Serum lactate.

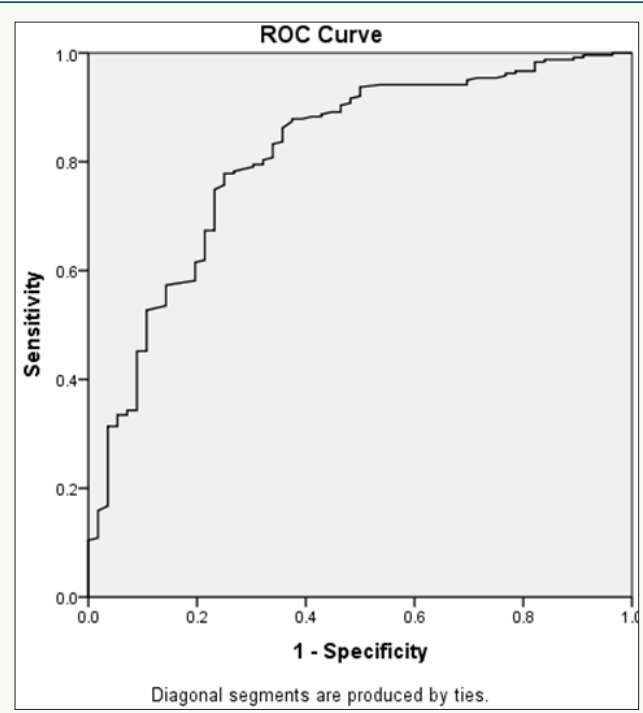

Figure 2: Cord arterial $\mathrm{pH}$.

Out of 295 neonates with abnormal CTG during labor, 32 neonates revealed severe metabolic acidosis (serum lactate $>10 \mathrm{mmol} / \mathrm{l}$ ). Out of 32 neonates with severe metabolic acidosis, 24 neonates needed resuscitation efforts. Figures 1-4 shows ROC for cord blood lactate levels and cord arterial $\mathrm{pH}$ measurements for the neonates with Apgar scoring less than 7 at 1 minute. Area under ROC curve for cord blood lactate is 0.917 (i.e test is excellent) in comparison to cord $\mathrm{pH}$ measurements which is 0.789 (i.e test is fair), so measuring cord blood lactate levels is considered an excellent test in comparison to umbilical cord arterial $\mathrm{pH}$.

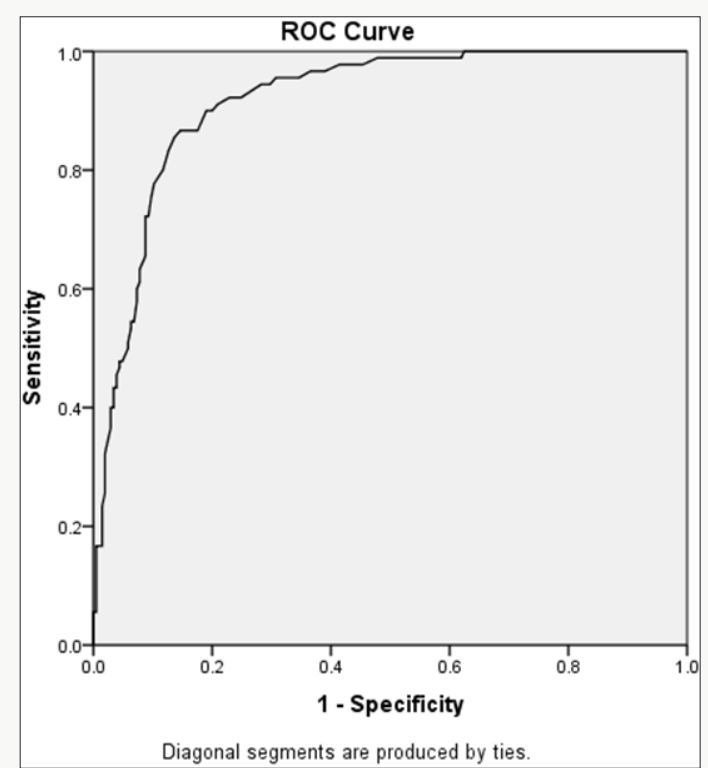

Figure 3: Serum lactate.

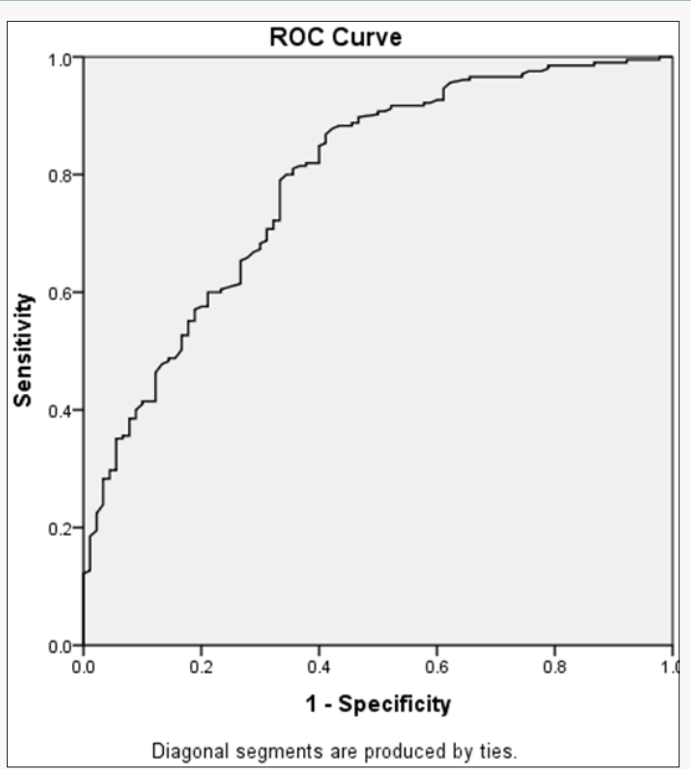

Figure 4: Cord arterial $\mathrm{pH}$.

\section{ROC for APGAR at 5 Minutes}

Out of 32 neonates presenting with severe metabolic acidosis, 22 neonates had Apgar scoring $\leq 7$ at 5 minutes. Figures 5 \& 6 displays ROC for umbilical cord blood lactate levels and cord arterial pH measurements for neonates with Apgar scoring less than 7 at 5 minutes. Area under ROC curve for cord blood lactate levels is 0.873 (i.e test is good) in comparison to cord $\mathrm{pH}$ measurements which is 0.786 (i.e test is fair), therefore cord blood lactate levels is a superior test in comparison to umbilical cord arterial $\mathrm{pH}$. 


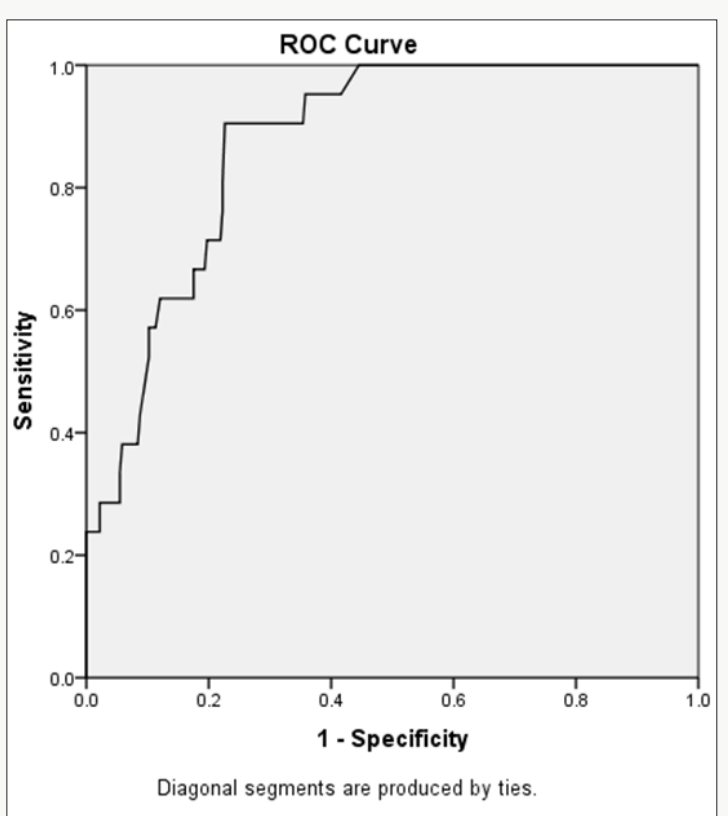

Figure 5: Cord blood lactate.

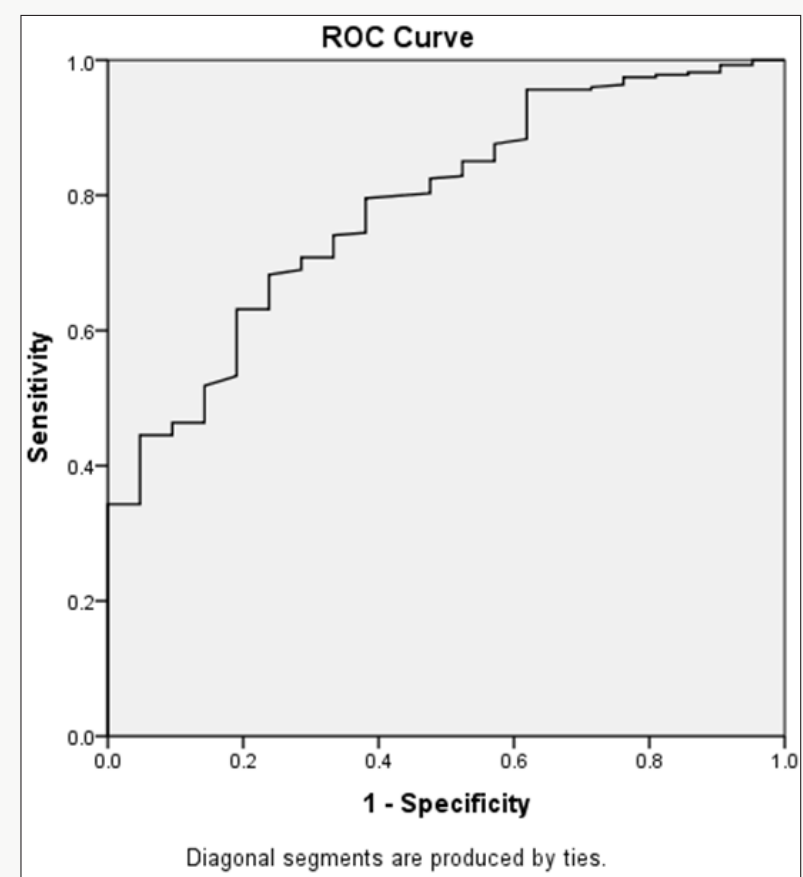

Figure 6: Cord arterial $\mathrm{pH}$.

\section{ROC for NICU Admission}

Out of 295 neonates, 21 neonates have been admitted to neonatal ICU. Figures $7 \& 8$ displays ROC for serum lactate levels and cord arterial $\mathrm{pH}$ measurements in neonates admitted to neonatal ICU. Area under ROC curve for cord blood lactate levels is 0.878 (i.e test is good) in comparison to cord $\mathrm{pH}$ measurements which is 0.817 (test is good), therefore umbilical cord blood lactate levels and cord arterial $\mathrm{pH}$ measurements both are fine in predictability parameters neonatal ICU Admission.

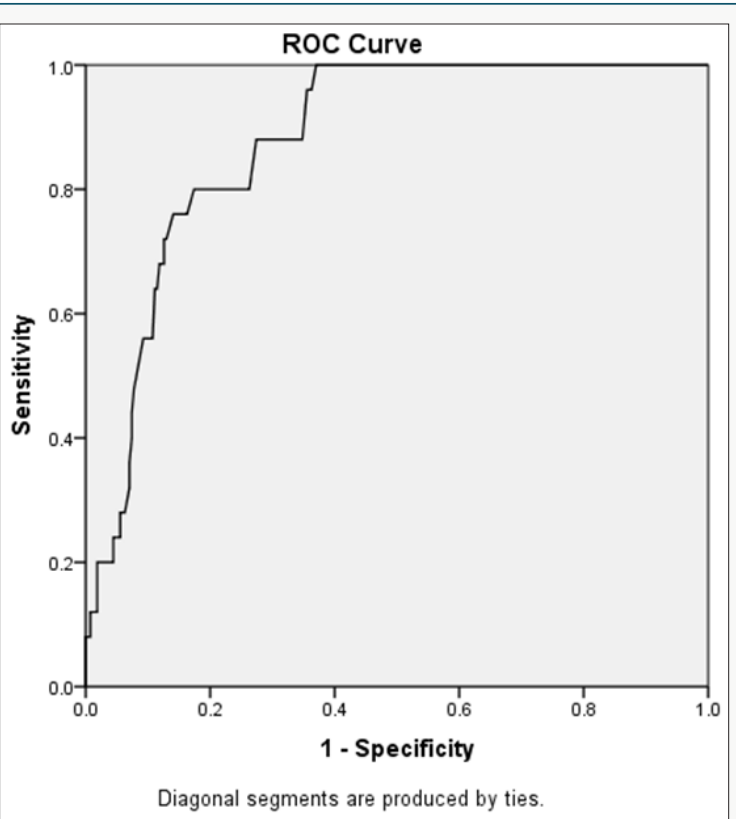

Figure 7: Cord blood lactate.

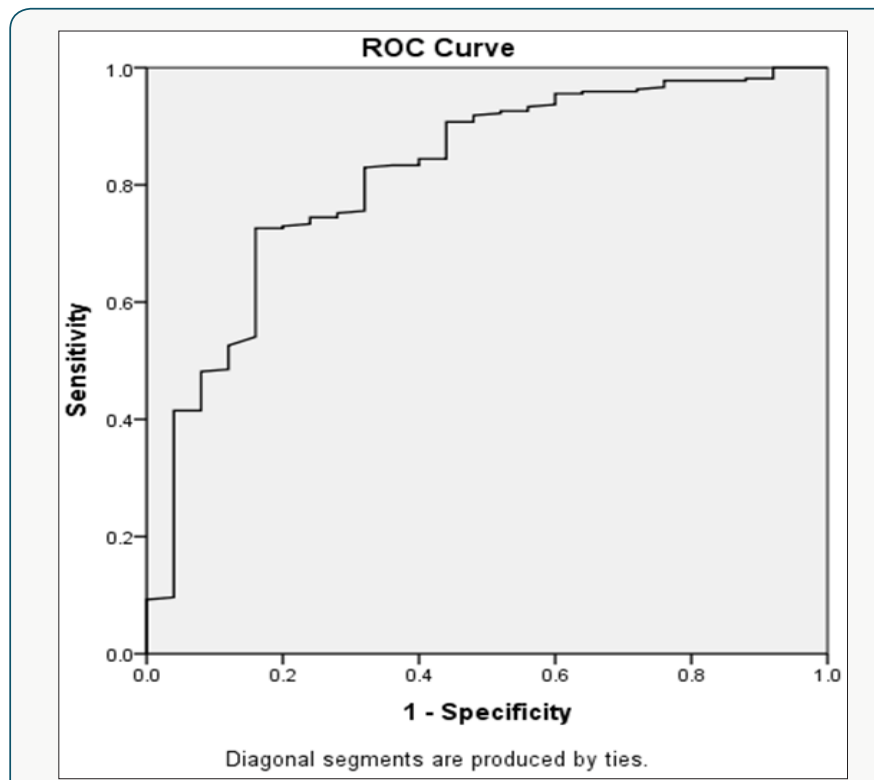

Figure 8: Cord arterial $\mathrm{pH}$.

\section{ROC for Unfavorable Neonatal Clinical Outcome}

Out of 295 neonates, 7 neonates developed convulsions within 24 hours of delivery. Out of 7 neonates, 5 neonates had serum lactate level $>10 \mathrm{mmol} / \mathrm{l}$. Figures $9 \& 10$ displays cord blood lactate levels and cord arterial $\mathrm{Ph}$ measurements in neonates with unfavorable obstetric clinical outcomes. Area under ROC curve for cord blood lactate level is 0.905 ( i.e test is excellent) in comparison to cord $\mathrm{pH}$ measurements which is 0.891 (i.e test is good), therefore cord blood lactate levels is an excellent tool in comparison to umbilical cord arterial $\mathrm{pH}$ in predictability unfavorable neonatal clinical outcomes. 


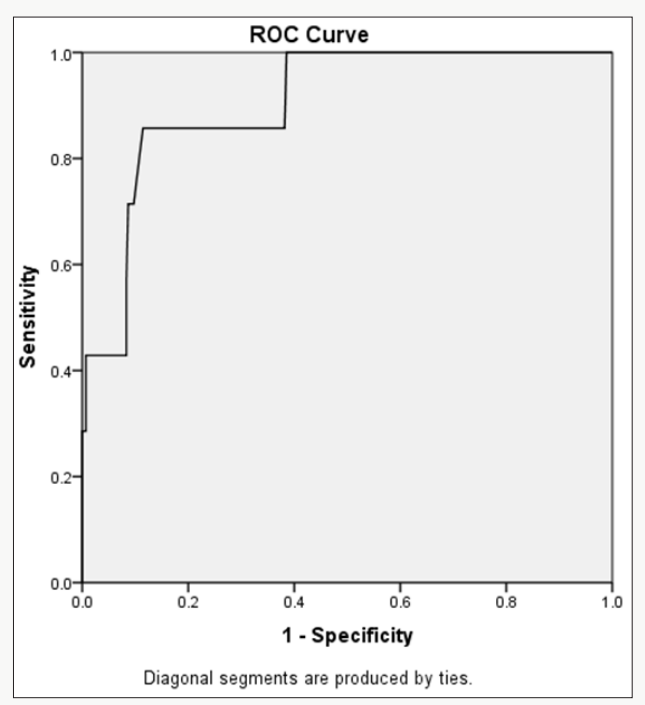

Figure 9: Serum lactate.

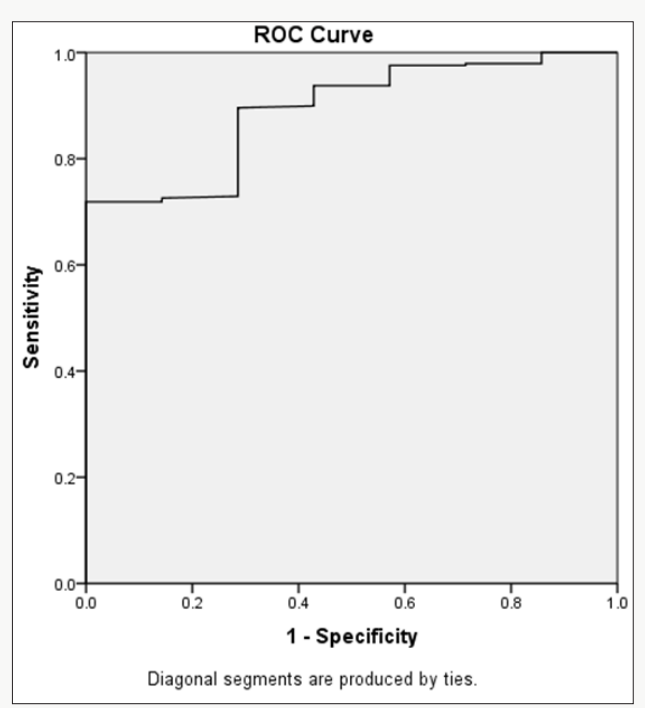

Figure 10: Cord arterial $\mathrm{pH}$.

\section{Discussion}

This is a research study conducted in an observational manner in which 295 gestations in labor with CTG trace abnormalities clinically suspected to comprise fetal distress within intra partum period have been recruited as study subjects. The research has been performed between 2015 to June 2017 in Demerdash University Hospital. A consent have been obtained from recruited term gestations with CTG trace abnormalities, umbilical cord blood was sampled and analyzed for lactate serum levels and umbilical cord $\mathrm{pH}$ measurements and both parameters were implemented for predictability of unfavorable neonatal clinical outcomes and consequently compared and contrasted. Neonates with both normal and abnormal serum lactate levels research study groups were revealed to be comparable as regards to maternal age, parity, neonatal birth weight and gender of the neonate therefore excluding the vast majority of the statistically confounding factors. Within the
295 neonates with CTG trace abnormalities, 32 neonates displayed profound metabolic acidosis (serum lactate levels $>10 \mathrm{mmol} / \mathrm{l}$ ). Within 32 neonates with profound metabolic acidosis, 28 neonates revealed clinically apgar scoring $\leq 7$ at 1 minute, 22 neonates clinically revealed Apgar scoring $\leq 7$ at 5 minutes, 24 neonates needed neonatal resuscitation efforts , 21 neonates were admitted to neonatal ICU and 5 neonates presented with convulsions within 24 hours of delivery. In the current research study neonates with Apgar scoring $\leq 7$ at 1 minute, $87.5 \%$ of the babies displayed raised umbilical cord blood lactate levels and Apgar scoring $\leq 7$ at 5 minutes only $68.7 \%$ of neonates displayed raised umbilical cord blood lactate measurements. Calculated specificity of serum lactate levels (97.7\%) and umbilical cord artery $\mathrm{pH}$ measurements (95.97\%) was nearly alike in neonates with clinical Apgar scoring of $\leq 7$ at $1 \mathrm{~min}$, however calculated sensitivity of serum lactate measurements (23.14\%) and cord pH levels (31.4\%) is less in neonates with clinical Apgar scoring $\leq 7$ at 1 minute after birth. The current research performed was similar to a research previously performed by the research group of Damodaran G et al. [4] in their study research approach on APGAR scoring and sampled umbilical cord blood measurements of lactate and Creatinine in clinical scenarios of Prenatal asphyxia came to a conclusion that umbilical cord blood lactate measurement of neonates will assist in clinical assessment of the severity of hypoxia [13-15].

In the current research study conducted area under ROC curve displayed that serum lactate levels is more precise and clinically useful in forecasting unfavorable neonatal clinical outcomes (Area under ROC for serum lactate levels was displayed to be between 0.8-0.9) in comparison to umbilical artery pH measurements (area under ROC for umbilical artery $\mathrm{p} \mathrm{H}$ was between $0.7-0.8$ ). In the current research study we implemented the 95th percentile as cut off parameter for serum lactate levels (i.e $10 \mathrm{mmol} / \mathrm{l}$ ). If we consider the 95th percentile $(10 \mathrm{mmol} / \mathrm{l})$ as cut off parameter, calculated specificity of serum lactate levels rises in comparison to umbilical cord arterial $\mathrm{pH}$ measurements in forecasting. Unfavorable neonatal clinical outcomes similar to reduced Apgar scoring. Resuscitation, neonatal ICU admission and hypoxic issues within the first 24 hours of birth. In comparison to a previously performed research in a descriptive approach manner of umbilical cord arterial blood analyzed samples from 2554 singleton births, ROC curves displayed and implied a lactate cut-off parameter of $8 \mathrm{mmol} / \mathrm{l}$ for reflection of intra partum asphyxia issues. 14 In the current research study ROC curves displayed and implied that measured lactate cut off measurement of $10 \mathrm{mmol} / \mathrm{l}$ for reflection of intra partum asphyxia issues.

Within 295 neonates 7 neonates developed convulsions within one day of delivery. From 7 neonates, 5 neonates had serum lactate level $>10 \mathrm{mmol} / \mathrm{l}$ showing that serum lactate is a superior predictability tool of hypoxic events in first 24 hours of delivery. Our research study in correlation with a previous research performed by Shah S et al. [16] But in the present study, we performed only 
one time measurement of serum lactate levels not sequential measurements for forecasting early neonatal clinical outcomes. On the other hand Shah S et al in his conducted research study on post natal lactate as an early tool for prediction of short-term clinical outcomes after occurrence of intra partum asphyxia issues, came to a conclusion that the maximum measured serum lactate level in the first hour of delivery and sequential measuring of lactate levels are significant clinical predictability tools of moderate-to-severe hypoxic ischemic encephalopathy [16-18]. Another researcher displayed that a correlation exist between the serum lactate levels and $\mathrm{pH} /$ base deficit in both the umbilical artery and vein in normal neonates as well as in clinical scenarios with fetal distress. Since lactate is much more simple and economic to analyze, it is a hopeful tool that could replace $\mathrm{pH}$ or base deficit measurement in evaluation of the metabolic condition of neonate [10-12].

\section{Restrictions of the Current Research Study}

Small recruited sample size, further research studies with more laboratory parameters are required putting in consideration ethnic and racial differences are required. Our current research study we have recruited gestations with CTG trace abnormalities and there is was no statistical comparison performed with gestations with normal CTG traces and its relation with measured serum lactate levels and umbilical cord $\mathrm{pH}$ parameters in forecasting unfavorable neonatal clinical outcomes. Lactate levels and umbilical artery $\mathrm{pH}$ measurements obtained on cord blood sample immediately after birth, the research group did not perform serial measurements of lactate levels and $\mathrm{pH}$ in correspondence to 1 minute and 5 minutes clinical APGAR scoring in forecasting unfavorable prenatal clinical outcomes.

\section{Conclusion}

WE concluded the following from our research study that umbilical cord blood lactate levels is a superior tool than umbilical artery $\mathrm{pH}$ in predictability of unfavorable neonatal outcome, as it represents the metabolic mirror of the acid-base physiological balance. Area under ROC (at 95th percentile) displayed that serum lactate is much more precise in predicting unfavorable neonatal clinical outcome in comparison to umbilical artery ph at delivery.

Conflict of interest: authors declare no conflict of interest

\section{References}

1. Malin GL, Morris RK, Kha KS (2010) Strength of association between umbilical cord $\mathrm{pH}$ and prenatal and long-term outcomes: systematic review and meta-analysis. BMJ 340: c1471.

2. Parveen S (2010) Umbilical cord arterial blood base excess as gold standard for foetal well-being screening test validity at term delivery. J Pak Med Assoc 60(5): 347-350.
3. Hamed HO (2013) Intrapartum fetal asphyxia: study of umbilical cord blood lactate in relation to fetal heart rate patterns. Arch Gynecol Obstet 287(6): 1067-1073.

4. Damodaran GK (2015) APGAR score and umbilical cord blood levels of lactate and creatinine in perinatal asphyxia. Int J Biomed Res 6(4): 242245.

5. Gjerris AC, Staer Jensen J, Jørgensen JS, Bergholt T, Nickelsen C (2008) Umbilical cord blood lactate: a valuable tool in the assessment of fetal metabolic acidosis. Eu J Obstet Gynecol Reprod Biol 139(1): 16-20.

6. Gillam Krakauer M, Carter BS (2012) Neonatal hypoxia and seizures. Pediatr Rev/Am Acad Pediatr 33(9): 387-396.

7. Alfirevic Z, Devane D, Gyte GM (2017) Continuous cardiotocography (CTG) as a form of electronic fetal monitoring (EFM) for fetal assessment during labor. Cochrane Database Syst Rev (5): CD006066.

8. Devane D, Lalor JG, Daly S, McGuire W, Smith V (2017) Cardiotocography versus intermittent auscultation of fetal heart on admission to labor ward for assessment of fetal wellbeing. Cochrane Database Syst Rev (1):CD005122.

9. Tranquilli AL (2012) Fetal heart rate in the second stage of labor: recording, reading, interpreting and acting. J Matern Fetal Neonatal Med 25(12): 2551-2554.

10. Allanson ER, Waqar T, White C, Tuncalp O, Dickinson JE (2017) Umbilical lactate as a measure of acidosis and predictor of neonatal risk: a systematic review. BJOG 124(4): 584-594.

11. White CR, Doherty DA, Newnham JP, Pennell CE (2014) The impact of introducing universal umbilical cord blood gas analysis and lactate measurement at delivery. Aust N Z J Obstet Gynaecol 154(1): 71-78.

12. Pattinson RCRN (2014) Saving babies 2012-2013: Ninth report on perinatal care in South Africa. Pretoria, South Africa.

13. Tuuli MG, Stout MJ, Macones GA, Cahill AG (2016) Umbilical Cord Venous Lactate for Predicting Arterial Lactic Acidemia and Neonatal Morbidity at Term. Obstetr Gynaecol 127(4): 674-680.

14. Dani C, Bresci C, Berti E, Lori S, Di Tommaso MR et al. (2013) Short term outcome of term newborns with unexpected umbilical cord arterial $\mathrm{pH}$ between 7.000 and 7.100. Early Hum Dev 89(12): 1037-1040.

15. White CR, Doherty DA, Henderson JJ, Roland Kohan, JP Newnham, et al. (2012)Accurate prediction of hypoxic-ischaemic encephalopathy at delivery: a cohort study. J Matern Fetal Neonatal Med 25: 1653-1659.

16. Tuuli MG, Stout MJ, Shanks A, Odibo AO, Macones GA et al. (2014) Umbilical cord arterial lactate compared with $\mathrm{pH}$ for predicting neonatal morbidity at term. Obstetr Gynecol 124(4): 756-761.

17. Zanardo V, Mari G, de Luca F, Scambia G, Guerrini P, et al. (2015) Lactate in cord blood and its relation to fetal gluconeogenesis in at term deliveries. Early Hum Dev 91(3): 165-168.

18. Heinis AM, Spaanderman ME, Gunnewiek JM, Lotgering FK (2011) Scalp blood lactate for intra-partum assessment of fetal metabolic acidosis. Acta Obstet Gynecol Scand 90(10): 1107-1114. 
(c) (i) This work is licensed under Creative

To Submit Your Article Click Here: $\quad$ Submit Article

DOI: 10.32474/IGWHC.2018.01.000123

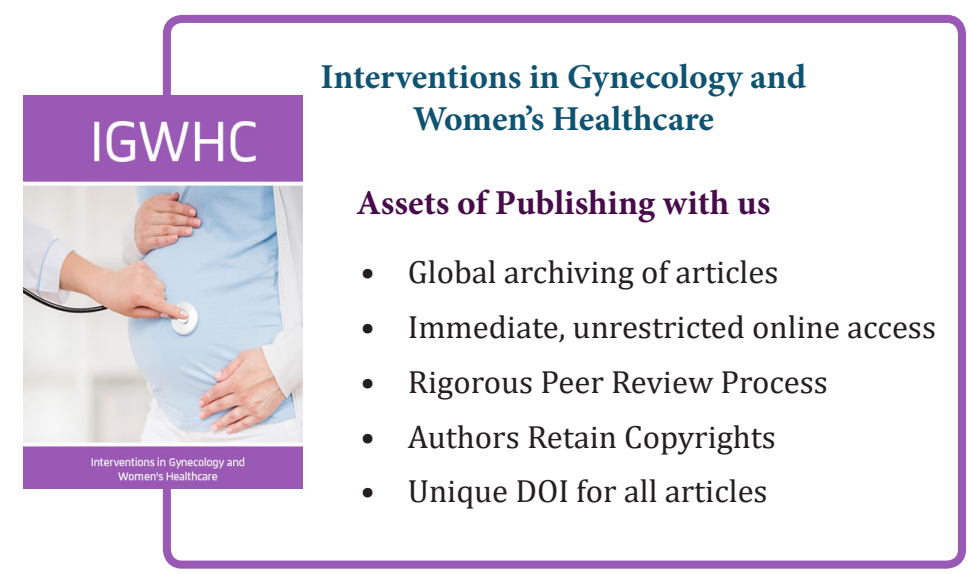

